J. Korean Math. Soc. 50 (2013), No. 5, pp. 1051-1066

http://dx.doi.org/10.4134/JKMS.2013.50.5.1051

\title{
INJECTIVE MODULES OVER $w$-NOETHERIAN RINGS, II
}

\author{
Jun Zhang, Fanggui Wang, and Hwankoo Kim
}

\begin{abstract}
By utilizing known characterizations of $w$-Noetherian rings in terms of injective modules, we give more characterizations of $w$-Noetherian rings. More precisely, we show that a commutative ring $R$ is $w$ Noetherian if and only if the direct limit of $G V$-torsion-free injective $R$ modules is injective; if and only if every $R$-module has a $G V$-torsion-free injective (pre)cover; if and only if the direct sum of injective envelopes of $w$-simple $R$-modules is injective; if and only if the essential extension of the direct sum of $G V$-torsion-free injective $R$-modules is the direct sum of $G V$-torsion-free injective $R$-modules; if and only if every $\mathscr{F}_{w, f}(R)$ injective $w$-module is injective; if and only if every GV-torsion-free $R$ module admits an $i$-decomposition.
\end{abstract}

\section{Introduction}

For the last few decades, characterizing Noetherian rings in terms of injective modules has drawn considerable attention from many algebraists. Matlis ([19]), Papp ([20]), Bass ([2]), Faith and Walker ([8]), Kurshan ([18]), Goursaud and Valette ([11]), Beidar and Ke ([4]), and Beidar, Jain and Srivastava ([3]) have done much meaningful work in this field. Since the birth of the theory of star operations, heavy concentration has been put on ideal theory. Even so, we still hope that the theory of star operations can play a role in researching the direct sum representations of injective modules and related topics [14]. Inspired by the study on injective modules over Noetherian rings, some researchers have paid attention to the studies on injective modules over $w$-Noetherian rings. In [26], Yin et al. defined a $w$-Noetherian ring as a commutative ring which satisfies the ascending chain condition of $w$-ideals. As for the integral domain, a $w$-Noetherian ring actually is a strong Mori domain. In 2005, Fuchs proved that the integral domain $R$ is a strong Mori domain if and only if $E(Q / R)$ is a $\Sigma$ injective module [9]. According to the Cartan-Eilenberg-Bass-Papp Theorem, $R$ is a Noetherian ring if and only if the direct sum of injective modules is injective. In 2008, Kim et al. proved that the integral domain $R$ is a strong

Received November 23, 2012; Revised February 26, 2013.

2010 Mathematics Subject Classification. Primary 13A15, 13C11, 13D99, 13 E99.

Key words and phrases. $G V$-torsion-free module, $w$-module, $w$-simple module, $w$ Noetherian ring, injective module. 
Mori domain if and only if the direct sum of co-semi-divisorial (namely, $G V$ torsion-free) injective modules is still injective [15]. In 2010, Wang and Zhang further proved that $R$ is a $w$-Noetherian ring if and only if the direct sum of $G V$ torsion-free injective $R$-modules is injective; if and only if every $G V$-torsion-free injective module is $\Sigma$-injective [25]. In 2011, Kim and Wang characterized $w$ Noetherian rings as the $w$-analogue of the Matlis-Papp Theorem [17], that is, $R$ is a $w$-Noetherian ring if and only if every $G V$-torsion-free injective module is the direct sum of indecomposable $G V$-torsion-free injective modules. These results not only deepened our understanding of characterizations of injective modules over $w$-Noetherian rings, but also of equivalent characterizations from the module-theoretic point of view.

It is well-known that $R$ is a Noetherian ring if and only if the direct limit of injective modules is injective. Thus a natural question is whether the $w$ theoretic analogue of this theorem holds true. For this purpose, we try to give much richer and more complete characterizations of $w$-Noetherian rings in terms of injective modules or of a weaker condition than injectivity.

In Section 2, we prove that $R$ is a $w$-Noetherian ring if and only if the direct limit of the $G V$-torsion-free injective modules is injective. The main challenge in proving this result is to prove isomorphism theorems about Ext functors. According to the classic homological theory, Ext functors work on exact sequences. As for a $w$-Noetherian ring, finite type and finitely presented type modules are defined through $w$-exact sequences [22], and Ext functors cannot work on $w$-exact sequences directly. However, inspired by [17] and [25], we work to find that the direct limit of $w$-modules over $w$-Noetherian rings is a $w$-module and to find other meaningful results. Many researchers are very interested in characterizing some classes of rings in terms of precovers and covers in ring and module theories. Based on the theorem mentioned above, we prove that $R$ is a $w$-Noetherian ring if and only if every $R$-module has a $G V$-torsion-free injective precover; if and only if every $R$-module has a $G V$ torsion-free injective cover.

From the works of Kurshan and Beidar-Ke, we can see that the direct sum of injective envelopes of simple modules plays an important role in characterizations of Noetherian rings. According to the Kurshan Theorem, $R$ is a Noetherian ring if and only if every countable direct sum of injective envelopes of simple modules is injective [18, Theorem 2.4]. Inspired by this result, in Section 3, we define $w$-simple modules and illustrate the difference between $w$ simple modules and simple modules. Through the new concept, we prove that $R$ is a $w$-Noetherian ring if and only if every countable direct sum of injective envelopes of $w$-simple $R$-modules is injective; if and only if every direct sum of injective envelopes of $w$-simple $R$-modules is injective. Moreover, we prove that $R$ is a $w$-Noetherian ring if and only if the essential extension of the direct sum of $G V$-torsion-free injective $R$-modules is the direct sum of $G V$-torsion-free injective modules; if and only if for each $G V$-torsion-free injective $R$-module $M$ and each index set $\Gamma$, every essential extension of $M^{(\Gamma)}$ is the direct sum of 
$G V$-torsion-free injective modules. This theorem extends the result in [25] by Wang and Zhang. Lastly, we give characterizations of $w$-Noetherian rings in terms of a weaker condition than injectivity and $i$-decomposition respectively. It is shown that $R$ is a $w$-Noetherian ring if and only if every $\mathscr{F}_{w, f}(R)$-injective $w$-module is injective; if and only if every GV-torsion-free $R$-module admits an $i$-decomposition.

Next we introduce some definitions and notations from [22] and [26]. Throughout this paper, we let $R$ be a commutative ring with identity. A finitely generated ideal $J$ of $R$ is called a $G V$-ideal if the natural homomorphism $R \rightarrow$ $J^{*}=\operatorname{Hom}_{R}(J, R)$ is an isomorphism. This definition is consistent with that in [23] when $R$ is a domain. Denote by $G V(R)$ the set of $G V$-ideals of $R$. It is proved in [26] that $G V(R)$ is a multiplicative system of ideals. Let $M$ be an $R$-module and define

$$
g v \cdot \operatorname{Tor}(M)=\{x \in M \mid J x=0 \text { for some } J \in G V(R)\} .
$$

Then $M$ is said to be $G V$-torsion (resp., $G V$-torsion-free) if $g v$. $\operatorname{Tor}(M)=M$ (resp., $g v$. $\operatorname{Tor}(M)=0$ ). It is known that a module $M$ is $G V$-torsion if and only if $\operatorname{Hom}_{R}(M, N)=0$ for any $G V$-torsion-free module $N$ [26, Theorem 1.4]. As in [22] and [26], a module $M$ is called a $w$-module if it has the following two properties: (1) $M$ is $G V$-torsion-free, (2) $\operatorname{Ext}_{R}^{1}(R / J, M)=0$ for each $J \in G V(R)$, or equivalently, $\operatorname{Ext}_{R}^{1}(N, M)=0$ for each $G V$-torsion module $N$. This is consistent with that in [23] when $R$ is a domain and $M$ is torsionfree. From the definition of $w$-modules, it is clear that every $G V$-torsion-free injective module is a $w$-module. Let $M$ be a $G V$-torsion-free module. In [26], the $w$-envelope of $M$ is defined by

$$
M_{w}=\{x \in E(M) \mid J x \subseteq M \text { for some } J \in G V(R)\},
$$

where $E(M)$ is the injective envelope of $M$. In [26], it is shown that for a $G V$-torsion-free $R$-module $M, M$ is a $w$-module if and only if $M_{w}=M$.

Refer to $[22,25,26]$ for more information on $w$-modules and $w$-Noetherian rings. For any unexplained terminologies and notations, we refer to $[5,7]$

\section{Direct limits of $G V$-torsion-free injective modules}

Firstly, we recall several concepts from [22]. A sequence $A \rightarrow B \rightarrow C$ is said to be $w$-exact if $A_{\mathfrak{m}} \rightarrow B_{\mathfrak{m}} \rightarrow C_{\mathfrak{m}}$ is exact for any maximal $w$-ideal $\mathfrak{m}$ of $R$. A homomorphism $f: M \rightarrow N$ is called a $w$-monomorphism (resp., w-epimorphism, $w$-isomorphism) if $f_{\mathfrak{m}}: M_{\mathfrak{m}} \rightarrow N_{\mathfrak{m}}$ a monomorphism (resp., epimorphism, isomorphism) for any maximal $w$-ideal $\mathfrak{m}$ of $R$. An $R$-module $M$ is said to be of finite type if there exists a $w$-exact sequence $F \rightarrow M \rightarrow 0$, while an $R$-module $M$ is said to be of finitely presented type if there exists a $w$-exact sequence $F_{1} \rightarrow F \rightarrow M \rightarrow 0$, where $F$ and $F_{1}$ are finitely generated and free. Denote by $\underset{\lim }{\longrightarrow} N_{i}$ the direct limit of a direct system $\left\{N_{i}, \varphi_{j}^{i}\right\}$ of $R$-modules. 
Lemma 2.1. Let $M$ be a finitely presented $R$-module. Then

$$
\underset{\lim _{\longrightarrow}}{ } \operatorname{Hom}_{R}\left(M, N_{i}\right) \cong \operatorname{Hom}_{R}\left(M, \underset{\lim }{\longrightarrow} N_{i}\right) \text {. }
$$

Proof. The proof is similar to that of [7, Lemma 3.1.16].

Proposition 2.2. Let $\left\{N_{i}\right\}$ be a family of $G V$-torsion-free $R$-modules. Then $\underset{\lim }{\longrightarrow} N_{i}$ is also $G V$-torsion-free.

Proof. Let $0 \rightarrow J \rightarrow R \rightarrow R / J \rightarrow 0$ be an exact sequence, where $J \in$ $G V(R)$. Then $R / J$ is finitely presented. By Lemma 2.1, for any $J \in G V(R)$, $\operatorname{Hom}_{R}\left(R / J, \underset{\lim }{\longrightarrow} N_{i}\right) \cong \operatorname{limHom}_{R}\left(R / J, N_{i}\right)=0$. Thus $\underset{\lim }{\longrightarrow} N_{i}$ is also $G V$-torsionfree.

Lemma 2.3. Let $\left\{N_{i}\right\}$ be a family of $G V$-torsion-free $R$-modules. If $M$ is a finite type $R$-module, then

$$
\tau: \underset{\lim }{\rightarrow} \operatorname{Hom}_{R}\left(M, N_{i}\right) \rightarrow \operatorname{Hom}_{R}\left(M, \lim _{\longrightarrow} N_{i}\right)
$$

is a monomorphism. In particular, if $M$ is finitely generated, then $\tau$ is a monomorphism.

Proof. Since $M$ is of finite type, $F \stackrel{f}{\rightarrow} M \rightarrow 0$ is $w$-exact, where $F$ is a finitely generated free module. Thus $F \rightarrow M \rightarrow M / \operatorname{Im} f \rightarrow 0$ is exact and $M / \operatorname{Im} f$ is $G V$-torsion. By Proposition 2.2, we have the following commutative diagram with exact rows:

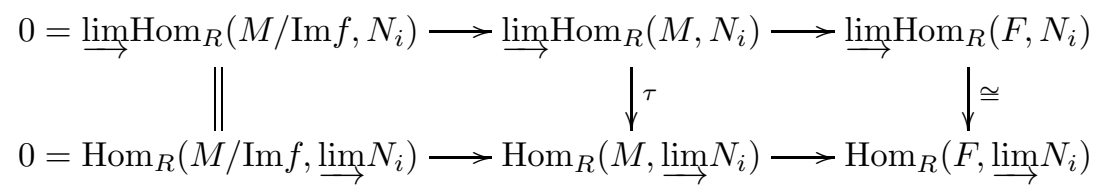

So $\tau$ is a monomorphism by the Five Lemma.

Theorem 2.4. Let $\left\{N_{i}\right\}$ be a family of $G V$-torsion-free $R$-modules. If $R$ is a $w$-Noetherian ring and $M$ is a finitely generated $R$-module, then

$$
\underset{\lim _{H}}{H_{0}}\left(M, N_{i}\right) \cong \operatorname{Hom}_{R}\left(M, \underset{\lim }{\longrightarrow} N_{i}\right) \text {. }
$$

Proof. If $M$ is finitely generated, then $0 \rightarrow K \rightarrow F \rightarrow M \rightarrow 0$ is exact, where $F$ is a finitely generated free module. Since $R$ is a $w$-Noetherian ring, $M$ is a finitely presented type module. Thus $K$ is of finite type. Then we have the following commutative diagram with exact rows:

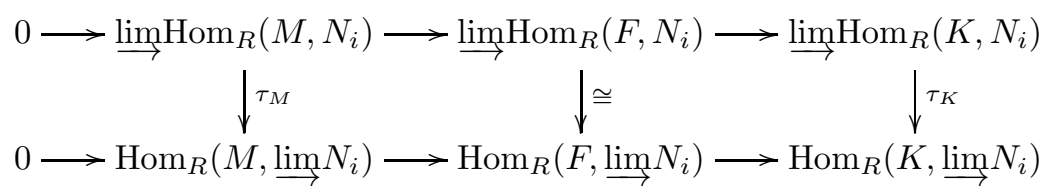

By Lemma 2.3, $\tau_{K}$ is a monomorphism. So by the Five Lemma, $\tau_{M}$ is an epimorphism. Therefore, $\tau_{M}$ is an isomorphism by Lemma 2.3. 
Proposition 2.5. Let $\left\{N_{i}\right\}$ be a family of $w$-modules. If $R$ is a $w$-Noetherian ring, then $\lim _{i} N_{i}$ is a w-module.

Proof. For any $J \in G V(R)$, there exists an exact sequence $0 \rightarrow J \rightarrow R \rightarrow$ $R / J \rightarrow 0$. Then we have the following commutative diagram with exact rows:

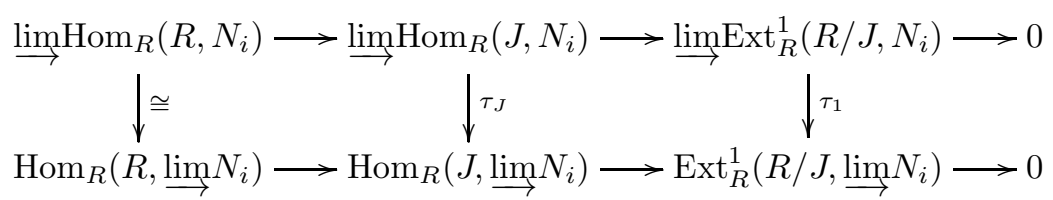

Since $J$ is finitely generated, $\tau_{J}$ is an isomorphism by Theorem 2.4. Thus $\tau_{1}$ is an isomorphism. Since each $N_{i}$ is a $w$-module, $\operatorname{Ext}_{R}^{1}\left(R / J, \underset{\lim }{\longrightarrow} N_{i}\right) \cong$ $\underset{\lim }{\longrightarrow} \operatorname{Ext}_{R}^{1}\left(R / J, N_{i}\right)=0$. Hence $\lim _{\longrightarrow} N_{i}$ is a $w$-module.

In [6, Proposition 2.2], it is shown that for modules $M$ and $N$ over an integral domain $R$, if $M$ is $G V$-torsion-free, then $\operatorname{Hom}_{R}\left(N_{w}, M_{w}\right) \cong \operatorname{Hom}_{R}\left(N, M_{w}\right)$. Although the following result is a special case of this result, we give a proof for the sake of completeness.

Proposition 2.6. Let $N$ be a $G V$-torsion-free $R$-module and let $M$ be a $w$ module. Then $\operatorname{Hom}_{R}(N, M) \cong \operatorname{Hom}_{R}\left(N_{w}, M\right)$.

Proof. From the exact sequence $0 \rightarrow N \rightarrow N_{w} \rightarrow N_{w} / N \rightarrow 0$, we have the long exact sequence $0 \rightarrow \operatorname{Hom}_{R}\left(N_{w} / N, M\right) \rightarrow \operatorname{Hom}_{R}\left(N_{w}, M\right) \rightarrow \operatorname{Hom}_{R}(N, M) \rightarrow$ $\operatorname{Ext}_{R}^{1}\left(N_{w} / N, M\right)$. Since $\operatorname{Hom}_{R}\left(N_{w} / N, M\right)=0$ and $\operatorname{Ext}_{R}^{1}\left(N_{w} / N, M\right)=0$, $\operatorname{Hom}_{R}(N, M) \cong \operatorname{Hom}_{R}\left(N_{w}, M\right)$.

Lemma 2.7. Let $\left\{N_{i}\right\}$ be a family of $w$-modules. If $R$ is a $w$-Noetherian ring and $I$ is a w-ideal, then $\operatorname{limExt}_{R}^{1}\left(R / I, N_{i}\right) \cong \operatorname{Ext}_{R}^{1}\left(R / I, \underline{\lim } N_{i}\right)$.

Proof. Consider the exact sequence $0 \rightarrow I \rightarrow R \rightarrow R / I \rightarrow 0$. We have the following commutative diagram with exact rows:

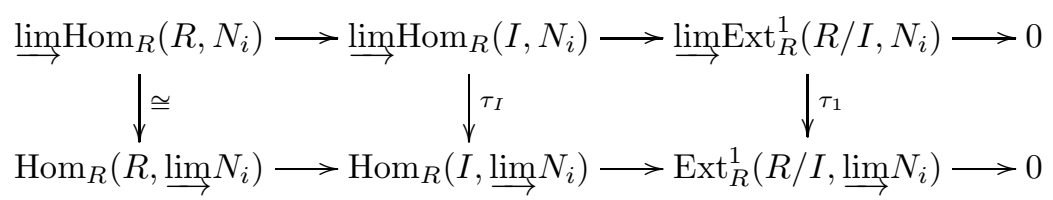

By hypothesis, $I$ is of finite type, so there exists a finitely generated subideal $B$ of $I$ such that $I=I_{w}=B_{w}$. By Theorem 2.4 and Proposition 2.6, we have $\underset{\longrightarrow}{\lim _{H}} \operatorname{mom}_{R}\left(I, N_{i}\right) \cong \lim _{\longrightarrow} \operatorname{Hom}_{R}\left(B_{w}, N_{i}\right) \cong \lim _{\longrightarrow} \operatorname{Hom}_{R}\left(B, N_{i}\right) \cong \operatorname{Hom}_{R}\left(B, \underset{\lim }{\longrightarrow} N_{i}\right)$ $\cong \operatorname{Hom}_{R}\left(B_{w}, \lim _{i}\right) \cong \operatorname{Hom}_{R}\left(I, \lim _{i} N_{i}\right)$. Therefore, $\tau_{1}$ is an isomorphism by the Five Lemma.

Lemma 2.8 ([17]). Let $E$ be a w-module. Then $E$ is injective if and only if $\operatorname{Ext}_{R}^{1}(R / I, E)=0$ for any $w$-ideal $I$ of $R$. 
It is shown in [5] that $R$ is a Noetherian ring if and only if the direct limit of injective modules is injective. Accordingly, we have the following theorem.

Theorem 2.9. The following statements are equivalent for a ring $R$ :

(1) $R$ is w-Noetherian;

(2) the direct limit of $G V$-torsion-free injective modules is injective;

(3) the direct sum of $G V$-torsion-free injective modules is injective.

Proof. $(1) \Rightarrow(2)$. Let $E=\lim _{\longrightarrow} E_{j}$, where each $E_{j}$ is a $G V$-torsion-free injective module, and $I$ be a $w$-ideal of $R$. By Proposition 2.5, Lemma 2.7 and Lemma 2.8, we have $\operatorname{Ext}_{R}^{1}(R / I, E) \cong \operatorname{limExt}_{R}^{1}\left(R / I, E_{i}\right)=0$. Thus $E$ is injective.

$(2) \Rightarrow(3)$. Since a direct sum is a direct limit of the finite sums, the direct sum of $G V$-torsion-free injective modules is injective.

$(3) \Rightarrow(1)$. [25, Theorem 4.4].

Let $M$ be an $R$-module. Then an injective cover of $M$ is a homomorphism $\phi: E \rightarrow M$ with $E$ injective such that

(1) for any injective $R$-module $E^{\prime}$ and any homomorphism $\phi^{\prime}: E^{\prime} \rightarrow M$, the diagram

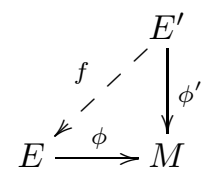

can be completed to a commutative diagram, i.e., there exists an $R$ module homomorphism $f: E^{\prime} \rightarrow E$ such that $\phi f=\phi^{\prime}$, and

(2) the diagram

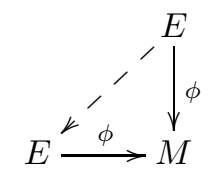

can only be completed by an automorphism of $E$.

Hence if an injective cover exists, then it is unique up to isomorphism. If $\phi$ : $E \rightarrow M$ satisfies (1), but perhaps not (2), then it is called an injective precover. It is well-known that a ring $R$ (not necessarily commutative) is Noetherian if and only if every $R$-module has an injective (pre)cover [7, Theorem 5.4.1]. The following result is the $w$-theoretic analogue of this seminal theorem.

Theorem 2.10. The following statements are equivalent for a ring $R$ :

(1) $R$ is $w$-Noetherian;

(2) every $R$-module has a $G V$-torsion-free injective precover;

(3) every $R$-module has a $G V$-torsion-free injective cover. 
Proof. $(1) \Rightarrow(2)$. Let $R$ be a $w$-Noetherian ring. By [25, Theorem 4.5], every $G V$-torsion-free injective $R$-module is a direct sum of indecomposable $G V$ torsion-free injective $R$-modules. Thus there is a family $\left\{E_{i} \mid i \in I\right\}$ of indecomposable $G V$-torsion-free injective $R$-modules such that every $G V$-torsion-free injective $R$-module is the direct sum of copies of the various $E_{i}$. Let $M$ be an $R$-module, $X_{i}=\operatorname{Hom}_{R}\left(E_{i}, M\right)$ and let $E_{i}^{\left(X_{i}\right)} \rightarrow M$ be the evaluation map defined by $\left(\varphi_{f}\right)_{f \in X_{i}} \mapsto \sum_{f \in X_{i}} f\left(\varphi_{f}\right)$. Then we have the following commutative diagram:

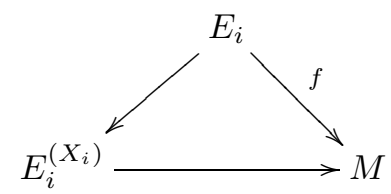

Thus, for any $G V$-torsion-free injective $R$-module $E$, we have the following commutative diagram:

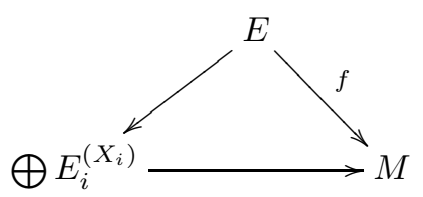

Therefore, $\bigoplus E_{i}^{\left(X_{i}\right)} \rightarrow M$ is a $G V$-torsion-free injective precover of $M$.

$(2) \Rightarrow(1)$. Let $\left\{E_{i} \mid i \in I\right\}$ be a family of $G V$-torsion-free injective $R$ modules. Then by hypothesis, $E \stackrel{\varphi}{\rightarrow} \bigoplus E_{i}$ is a $G V$-torsion-free injective precover of $\bigoplus E_{i}$, where $E$ is a $G V$-torsion-free injective module. For each $j \in I$, $E_{j} \stackrel{\lambda_{j}}{\rightarrow} \bigoplus E_{i}$ is the canonical injection, we have the following commutative diagram:

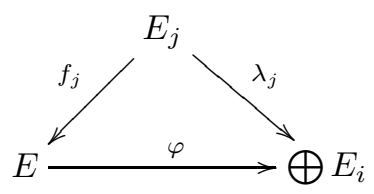

that is, $\varphi f_{j}=\lambda_{j}$. Hence we have the following commutative diagram:

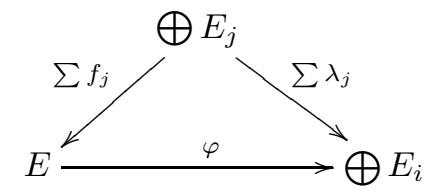

that is, $\varphi\left(\sum f_{j}\right)=\sum\left(\varphi f_{j}\right)=\sum \lambda_{j}=1$. So $\bigoplus E_{i}$ is isomorphic to a summand of $E$. Hence $\bigoplus E_{i}$ is a $G V$-torsion-free injective module. By [25, Theorem 4.4], $R$ is a $w$-Noetherian ring. 
$(2) \Rightarrow(3)$. Let $R$ be a $w$-Noetherian ring. By Theorem $2.9(2)$, the direct limit of $G V$-torsion-free injective $R$-modules is injective. Thus by [7, Corollary 5.2 .7 , every $R$-module has a $G V$-torsion-free injective cover.

$(3) \Rightarrow(2)$. This is clear.

Corollary 2.11. Let $R$ be a Noetherian ring. Then

(1) every $R$-module has a $G V$-torsion-free injective precover;

(2) every $R$-module has a $G V$-torsion-free injective cover.

Proposition 2.12. Let $M$ be a $G V$-torsion-free module. If $E$ is an essential extension of $M$, then $E$ is also $G V$-torsion-free.

Proof. Let $x \in E$ and $J \in G V(R)$ with $J x=0$. Assume $x \neq 0$. By hypothesis, there exists $r \in R$ such that $r x \neq 0$ and $r x \in M$. Since $M$ is $G V$-torsion-free and $J r x=0$, we have $r x=0$, a contradiction. Hence $E$ is $G V$-torsion-free.

Theorem 2.13. The following statements are equivalent for a ring $R$ :

(1) $R$ is w-Noetherian;

(2) the direct sum of $G V$-torsion-free injective modules is injective;

(3) if $\left\{M_{i}\right\}$ is a family of $G V$-torsion-free $R$-modules, then

$$
E\left(\bigoplus_{i \in I} M_{i}\right) \subseteq \bigoplus_{i \in I} E\left(M_{i}\right)
$$

(4) if $\left\{M_{i}\right\}$ is a family of $G V$-torsion-free $R$-modules, then

$$
E\left(\bigoplus_{i \in I} M_{i}\right)=\bigoplus_{i \in I} E\left(M_{i}\right)
$$

Proof. $(1) \Leftrightarrow(2)$. [25, Theorem 4.4].

$(2) \Rightarrow(3)$. Since $E\left(\bigoplus_{i \in I} M_{i}\right)$ is the injective envelope of $\bigoplus_{i \in I} M_{i}$, there is no other injective module between $\bigoplus_{i \in I} M_{i}$ and $E\left(\bigoplus_{i \in I} M_{i}\right)$. Since each $M_{i}$ is $G V$-torsion-free, $E\left(M_{i}\right)$ is also $G V$-torsion-free by Proposition 2.12. Hence $\bigoplus_{i \in I} E\left(M_{i}\right)$ is injective. Also, by $\bigoplus_{i \in I} M_{i} \subseteq \bigoplus_{i \in I} E\left(M_{i}\right)$, we have

$$
E\left(\bigoplus_{i \in I} M_{i}\right) \subseteq \bigoplus_{i \in I} E\left(M_{i}\right) \text {. }
$$

$(3) \Rightarrow(4)$. This is clear.

$(4) \Rightarrow(2)$. Let $\left\{E_{j}\right\}_{j \in J}$ be a family of $G V$-torsion-free injective $R$-modules. Then $\bigoplus_{j \in J} E_{j}=\bigoplus_{j \in J} E\left(E_{j}\right)=E\left(\bigoplus_{j \in J} E_{j}\right)$. Therefore, the direct sum of $G V$-torsion-free injective modules is injective.

\section{3. $w$-simplicity and injective modules}

From [4] and [18], we see that simple modules play an important role in characterizing Noetherian rings. Now we introduce the concept of $w$-simple modules. It is necessary to realize that simple modules and $w$-simple modules are two mutually exclusive concepts. 
Definition. Let $M$ be a nonzero $w$-module. $M$ is said to be $w$-simple if $M$ has no nontrivial $w$-submodule.

Clearly, a $w$-simple module is indecomposable.

Proposition 3.1. Let $M$ be a w-module. Then $M$ is $w$-simple if and only if $M=(R x)_{w}$ for any nonzero element $x \in M$.

Proof. Let $M$ be a $w$-simple module. For any $x \in M, x \neq 0$, we have that $(R x)_{w}$ is a nonzero $w$-submodule of $M$. Since $M$ has no nontrivial $w$ submodule, $M=(R x)_{w}$.

Conversely, suppose $M$ is not $w$-simple. Then there exists a nontrivial $w$ submodule $N$ of $M$. For any $x \in N, x \neq 0$, we have $(R x)_{w} \subseteq N \subset M$, that is, $(R x)_{w} \neq M$, a contradiction. Therefore, $M$ is $w$-simple.

It is clear that a $w$-simple module may also not be a cyclic $w$-module.

Proposition 3.2. Let $M$ be a w-module and let $N$ be a $w$-submodule of $M$. Then $N$ is a maximal $w$-submodule of $M$ if and only if $(M / N)_{w}$ is w-simple.

Proof. By [26, Theorem 2.7], $M / N$ is $G V$-torsion-free. Suppose $(M / N)_{w}$ is $w$ simple. If $N_{1}$ is a $w$-submodule of $M$ containing $N$ properly, then $\left(N_{1} / N\right)_{w}=$ $(M / N)_{w}$. So for any $x \in M$, there exists $J \in G V(R)$ such that $J \bar{x} \subseteq N_{1} / N$. Therefore, $J x \subseteq N_{1}$, whence $x \in N_{1}$. So $N_{1}=M$, and hence $N$ is a maximal $w$-submodule of $M$.

Conversely, suppose $N$ is a maximal $w$-submodule of $M$. If $B$ is a nonzero $w$ submodule of $(M / N)_{w}$, then set $A=B \bigcap(M / N)$. Then $A_{w}=B \bigcap(M / N)_{w}=$ $B$. Write $A=N_{1} / N$, where $N_{1}$ is a submodule of $M$ containing $N$ properly. Then $\left(N_{1}\right)_{w}=M$. Then for any $x \in M$, there exists $J \in G V(R)$ such that $J x \subseteq N_{1}$. Hence $J \bar{x} \subseteq A$. Then $A_{w}=B=(M / N)_{w}$, whence $(M / N)_{w}$ is $w$-simple.

Corollary 3.3. Let $M$ be a w-simple module. Then for any nonzero element $x \in M, \operatorname{ann}(x)$ is a maximal $w$-ideal of $R$.

Let $D$ be a domain. In the following, we denote by $Q(D)$ the quotient field of $D$.

Proposition 3.4. Let $\mathfrak{m}$ be a maximal $w$-ideal of $R$. Then $(R / \mathfrak{m})_{w}=Q(R / \mathfrak{m})$ $=R_{\mathfrak{m}} / \mathfrak{m} R_{\mathfrak{m}}$ is a w-simple module.

Proof. Let $x \in(R / \mathfrak{m})_{w} \backslash\{0\}$. Then $J x \subseteq R / \mathfrak{m}$ for some $J \in G V(R)$. Thus $\mathfrak{m} J x=0$, whence $\mathfrak{m} J \subseteq \operatorname{ann}(x)$. Since $\operatorname{ann}(x)$ is a $w$-ideal, $\mathfrak{m} \subseteq \operatorname{ann}(x)$. So $\operatorname{ann}(x)=\mathfrak{m}$ since $\mathfrak{m}$ is a maximal $w$-ideal. Hence $(R / \mathfrak{m})_{w}$ is an $R / \mathfrak{m}$-module. Since $J \nsubseteq \mathfrak{m}$, there exists $r \in J$ with $r \notin \mathfrak{m}$. Thus $\bar{r} \neq 0$ in $R / \mathfrak{m}$ and $\bar{r} x=r x \neq 0$. Therefore, $(R / \mathfrak{m})_{w}$ is an essential extension of $R / \mathfrak{m}$, whence we have $(R / \mathfrak{m})_{w} \subseteq Q(R / \mathfrak{m})$. Now let $x=\frac{r}{s}+\mathfrak{m} R_{\mathfrak{m}} \in Q(R / \mathfrak{m})$, where $r, s \in R$, $s \notin \mathfrak{m}$. Then $(\mathfrak{m}+R s) x \subseteq R / \mathfrak{m}$. Since $(\mathfrak{m}+R s)_{w}=R$, there exists $J \in G V(R)$ with $J \subseteq \mathfrak{m}+R s$. So $J x \subseteq R / \mathfrak{m}$. Hence $Q(R / \mathfrak{m}) \subseteq(R / \mathfrak{m})_{w}$. Then by Proposition 3.2, we have $(R / \mathfrak{m})_{w}=Q(R / \mathfrak{m})=R_{\mathfrak{m}} / \mathfrak{m} R_{\mathfrak{m}}$ is $w$-simple. 
Lemma $3.5([22])$. Let $M$ and $N$ be $G V$-torsion-free modules and let $f: M \rightarrow$ $N$ be a w-isomorphism. If $g: M_{w} \rightarrow N_{w}$ is the extension of $f$, then $g$ is an isomorphism.

Theorem 3.6. Let $M$ be a w-module. Then $M$ is $w$-simple if and only if there exists a maximal $w$-ideal $\mathfrak{m}$ of $R$ such that $M \cong(R / \mathfrak{m})_{w}$.

Proof. If $M \cong(R / \mathfrak{m})_{w}$, where $\mathfrak{m}$ is a maximal $w$-ideal of $R$, then $M$ is $w$-simple by Proposition 3.4.

Conversely, suppose $M$ is $w$-simple. Let $x \in M \backslash\{0\}$. Then $M=(R x)_{w}$ by Proposition 3.1. Since $\mathfrak{m}:=\operatorname{ann}(x)$ is a maximal $w$-ideal of $R$ by Corollary 3.3 and $R x \cong R / \mathfrak{m}, M \cong(R / \mathfrak{m})_{w}$ by Lemma 3.5 .

Example 3.7. Let $\mathfrak{m}$ be a maximal ideal of $R$ but not a maximal $w$-ideal. Then $M:=R / \mathfrak{m}$ is simple but not $G V$-torsion-free. Hence a simple module is not necessarily $w$-simple. Let $R:=F[X, Y]$ be the polynomial ring in two indeterminates over a field $F$ and let $F(Y)$ be the quotient field of $F[Y]$. Then $\mathfrak{m}:=(X)$ is a maximal $w$-ideal of $R$ but not a maximal ideal. Thus $N:=$ $R / \mathfrak{m} \cong F[Y]$ and $M:=N_{w}=F(Y)$ is $w$-simple by Proposition 3.4, but not simple. Therefore, a $w$-simple module is not necessarily simple.

Theorem 3.8. Let $M$ be a $w$-module. If $M$ is of finite type, then there exists a maximal $w$-submodule of $M$.

Proof. Let $M=\left(x_{1}, \ldots, x_{n}\right)_{w},\left(x_{1}, \ldots, x_{n-1}\right)_{w} \neq M$. Set

$\mathscr{S}=\left\{N \mid N\right.$ is a $w$-submodule of $\left.M, x_{1}, \ldots, x_{n-1} \in N, x_{n} \notin N\right\}$.

By Zorn's Lemma, $\mathscr{S}$ has a maximal element $P$, whence $P$ is a maximal $w$ submodule of $M$.

Lemma 3.9. Let $M$ be a $G V$-torsion-free module and let $N$ be a submodule of $M$. If $M / N$ is $G V$-torsion-free, then $(M / N)_{w} \cong\left(M_{w} / N_{w}\right)_{w}$.

Proof. Since the canonical homomorphism $M / N \rightarrow M_{w} / N_{w}$ is a $w$-isomorphism, $(M / N)_{w} \cong\left(M_{w} / N_{w}\right)_{w}$.

Lemma 3.10. Let $M$ be a $w$-module and let $N$ be a $w$-submodule of $M$. Then for any nonzero $w$-submodule $A$ of $(M / N)_{w}$, there is a $w$-submodule $K$ of $M$ containing $N$ properly such that $(K / N)_{w}=A$.

Proof. Let $A$ be a nonzero $w$-submodule of $(M / N)_{w}$. Set $B=A \cap(M / N)=$ $C / N$, where $C$ is a submodule of $M$ containing $N$ properly. By Lemma 3.9, $B_{w}=A_{w} \cap(M / N)_{w}=A \cap(M / N)_{w}=A=(C / N)_{w}=\left(C_{w} / N\right)_{w}$. Set $K=C_{w}$. Then the conclusion follows.

In [18], according to the Kurshan Theorem, $R$ is a Noetherian ring if and only if every countable direct sum of injective envelopes of simple modules is injective. For $w$-Noetherian rings, we generalize the Kurshan Theorem.

Theorem 3.11. The following statements are equivalent for a ring $R$ : 
(1) $R$ is w-Noetherian;

(2) the direct sum of $G V$-torsion-free injective modules is injective;

(3) the direct sum of injective envelopes of $w$-simple modules is injective;

(4) the countable direct sum of injective envelopes of $w$-simple modules is injective.

Proof. $(1) \Leftrightarrow(2)$. [25, Theorem 4.4].

$(2) \Rightarrow(3) \Rightarrow(4)$. This is clear.

$(4) \Rightarrow(1)$. Suppose $R$ is not a $w$-Noetherian ring. Then there exists a strictly ascending chain of $w$-ideals of $R$

$$
I_{1} \subset I_{2} \subset \cdots \subset I_{n} \subset \cdots .
$$

Set $I=\bigcup_{i} I_{i}$. Then $I$ is also a $w$-ideal and for any $a \in I$, there exists $i_{a} \in \mathbb{N}$ such that $a \in I_{i}$ for all $i \geqslant i_{a}$. For each $n \in \mathbb{N}$, choose $a_{n} \in I \backslash I_{n}$. By Theorem 3.8 and Lemma 3.10 , there is a maximal $w$-submodule $\left(A_{n} / I_{n}\right)_{w}$ of $\left(\left(R a_{n}+I_{n}\right)_{w} / I_{n}\right)_{w}$, where $A_{n}$ is a $w$-ideal containing $I_{n}$. By Proposition 3.2, $E_{n}:=\left(\left(\left(R a_{n}+I_{n}\right)_{w} / I_{n}\right)_{w} /\left(A_{n} / I_{n}\right)_{w}\right)_{w}$ is $w$-simple. Let $E\left(E_{n}\right)$ be the injective envelopes of $E_{n}$. We have the following commutative diagram:

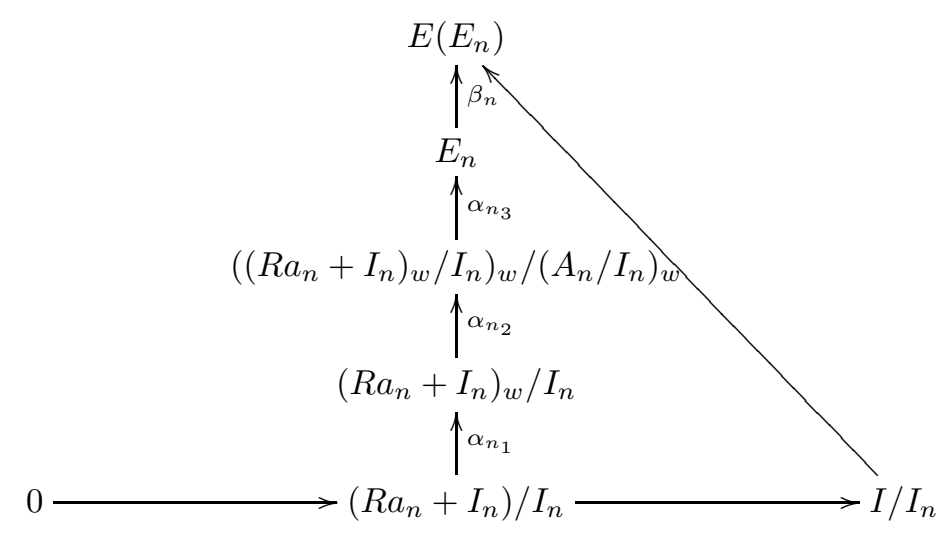

Set $\pi_{n}: I / I_{n} \rightarrow E\left(E_{n}\right)$ and $\alpha_{n}=\alpha_{n_{3}} \alpha_{n_{2}} \alpha_{n_{1}}$. Then $\pi_{n}\left(\bar{a}_{n}\right)=\beta_{n} \alpha_{n}\left(\bar{a}_{n}\right) \neq 0$. For any $n \in \mathbb{N}$, let $f: I \rightarrow \bigoplus_{n=1}^{\infty} E\left(E_{n}\right)$ be a homomorphism defined by

$$
f(a)=\left(\pi_{1}\left(a+I_{1}\right), \pi_{2}\left(a+I_{2}\right), \ldots, \pi_{n}\left(a+I_{n}\right), \ldots\right),
$$

where if $n \geqslant i_{a}$, then $\pi_{n}\left(a+I_{n}\right)=0$. By hypothesis, we have that $\bigoplus_{n=1}^{\infty} E\left(E_{n}\right)$ is injective. Thus we have the following commutative diagram:

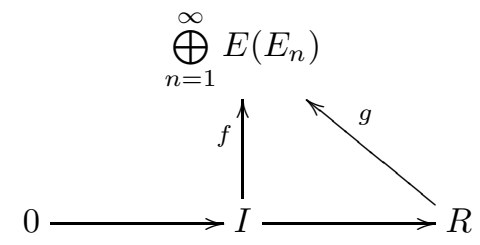


Set

$$
g(1)=\left(x_{1}, x_{2}, \ldots, x_{n}, \ldots\right), \quad x_{i} \in E\left(E_{i}\right),
$$

and $x_{n}=0$ for any $n \geqslant m$. Then for $t>m, f\left(a_{t}\right)=\left(\pi_{1}\left(a_{t}+I_{1}\right), \pi_{2}\left(a_{t}+\right.\right.$ $\left.\left.I_{2}\right), \ldots, \pi_{t}\left(a_{t}+I_{t}\right), \ldots\right)=g\left(a_{t}\right)=a_{t} g(1)=\left(a_{t} x_{1}, a_{t} x_{2}, \ldots, a_{t} x_{m}, 0, \ldots\right)$, and hence we have $\pi_{t}\left(a_{t}+I_{t}\right)=0$, a contradiction. Therefore, $R$ is $w$-Noetherian.

Recall from [10] that an injective module is $\Sigma$-injective if an infinite direct sum of its copies is injective. Following [21], we say that a module $M$ is finitely injective if every finite set of elements of $M$ is contained in an injective submodule.

Lemma $3.12([1,3])$. Let $M$ be an injective module. Then the following statements are equivalent:

(1) $M$ is a $\Sigma$-injective module;

(2) every essential extension of $M^{\left(\aleph_{0}\right)}$ is a direct sum of injective modules;

(3) every finitely injective submodule of $E\left(M^{\left(\aleph_{0}\right)}\right)$ is injective;

(4) every finitely injective submodule of $E\left(M^{\left(\aleph_{0}\right)}\right)$ is a direct sum of injective modules.

It is shown in $[1,3,4]$ that $R$ is a Noetherian ring if and only if every injective module is $\Sigma$-injective if and only if the essential extension of the direct sum of injective modules is the direct sum of injective modules if and only if for each injective module $M$, every essential extension of $M^{\left(\aleph_{0}\right)}$ is the direct sum of injective modules if and only if for each injective module $M$, every finitely injective submodule of $E\left(M^{\left(\aleph_{0}\right)}\right)$ is a direct sum of injective modules. The above results generalize the Cartan-Eilenberg-Bass-Papp Theorem for Noetherian rings. In [25], the authors proved the $w$-theoretic analogue of the Cartan-Eilenberg-BassPapp Theorem for Noetherian rings, that is, $R$ is a $w$-Noetherian ring if and only if the direct sum of $G V$-torsion-free injective modules is injective. Next we follow from Lemma 3.12 to easily obtain the following generalization of this theorem.

Theorem 3.13. The following statements are equivalent for a ring $R$ :

(1) $R$ is w-Noetherian;

(2) the direct sum of $G V$-torsion-free injective modules is injective;

(3) every $G V$-torsion-free injective module is $\Sigma$-injective;

(4) the essential extension of the direct sum of $G V$-torsion-free injective modules is the direct sum of $G V$-torsion-free injective modules;

(5) the essential extension of the countable direct sum of $G V$-torsion-free injective modules is the direct sum of $G V$-torsion-free injective modules;

(6) for each $G V$-torsion-free injective module $M$ and each index set $\Gamma$, every essential extension of $M^{(\Gamma)}$ is the direct sum of $G V$-torsion-free injective modules; 
(7) for each $G V$-torsion-free injective module $M$, every essential extension of $M^{\left(\aleph_{0}\right)}$ is the direct sum of $G V$-torsion-free injective modules;

(8) for each $G V$-torsion-free injective module $M$, every finitely injective submodule of $E\left(M^{\left(\aleph_{0}\right)}\right)$ is injective;

(9) for each $G V$-torsion-free injective module $M$, every finitely injective submodule of $E\left(M^{\left(\aleph_{0}\right)}\right)$ is a direct sum of injective modules.

Next we give another characterization of $w$-Noetherian rings in terms of a weaker condition than injectivity. To do so, we need some terminologies.

Let $\mathscr{F}_{w, f}(R)$ (resp., $\mathscr{F}_{w}(R)$ ) be the set of all finite type $w$-ideals (resp., $w$ ideals) of $R$. An $R$-module $M$ is said to be $\mathscr{F}_{w, f}(R)\left(\right.$ resp., $\left.\mathscr{F}_{w}(R)\right)$-injective if for every ideal $I \in \mathscr{F}_{w, f}(R)$ (resp., $\mathscr{F}_{w}(R)$ ), every $R$-homomorphism from $I$ into $M$ can be extended to an $R$-homomorphism from $R$ into $M$.

Example 3.14 (An example of a $G V$-torsion-free $\mathscr{F}_{w, f}(R)$-injective module that is not a $w$-module). Let $R:=F[X, Y]$ be the polynomial ring in two indeterminates over a field $F$ and let $Q:=F(X, Y)$ be its field of quotients. We consider the module $M:=Q / R$. It is shown in [16, Example 2.3] that $M$ is a divisible $R$-module that is not injective. It is also shown from [14, Corollary 2.11] that $M$ is a $G V$-torsion-free $R$-module. Note that $R$ is a factorial domain. Thus by [10, Lemma I.7.2], the $\mathscr{F}_{w}(R)$-injectivity (and hence $\mathscr{F}_{w, f}(R)$-injectivity) is equivalent to the divisibility. Now assume that $M$ is a $w$-module. Then by [17, Corollary 2.4], $M$ is injective, which is a contradiction. From this example, we can see easily that $\mathscr{F}_{w, f}(R)$-injective modules and injective modules are different.

Theorem 3.15. Let $\left\{M_{i}\right\}_{i \in I}$ be a family of $R$-modules. Then $\prod_{i \in I} M_{i}$ is $\mathscr{F}_{w, f}(R)$-injective if and only if each $M_{i}$ is $\mathscr{F}_{w, f}(R)$-injective.

Proof. The proof of the assertion is analogous to that of injectivity.

Theorem 3.16. Let $\left\{M_{i}\right\}_{i \in I}$ be a family of $w$-modules over $R$. Then $\bigoplus_{i \in I} M_{i}$ is $\mathscr{F}_{w, f}(R)$-injective if and only if each $M_{i}$ is $\mathscr{F}_{w, f}(R)$-injective.

Proof. Suppose that each $M_{i}$ is $\mathscr{F}_{w, f}(R)$-injective. Let $A=\left(x_{1}, x_{2}, \ldots, x_{n}\right)_{w}$ be a finite type $w$-ideal of $R$ and let $f: A \rightarrow \bigoplus_{i \in I} M_{i}$ be a homomorphism. Then $f(A) \subseteq\left(\bigoplus_{j \in J} M_{j}\right)_{w}=\bigoplus_{j \in J}\left(M_{j}\right)_{w}=\bigoplus_{j \in J} M_{j}$ for some finite subset $J$ of $I$. The remainder of this assertion follows from Theorem 3.15. The proof of the converse is similar to that of Theorem 3.15.

In [12], it is shown that $R$ is a Noetherian ring if and only if $f(R)$-injectivity is equivalent to injectivity, where $f(R)$ is the set of all finitely generated ideals of $R$. In the following, we get the $w$-analogue of this result.

Theorem 3.17. A ring $R$ is $w$-Noetherian if and only if every $\mathscr{F}_{w, f}(R)$ injective $w$-module is injective. 
Proof. Suppose that $R$ is $w$-Noetherian and let $M$ be an $\mathscr{F}_{w, f}(R)$-injective $w$ module. Then in order to prove that $M$ is injective, by Lemma 2.8 , it suffices to show that $M$ is $\mathscr{F}_{w}(R)$-injective. But this follows from the facts that $R$ is $w$-Noetherian and that $M$ is $\mathscr{F}_{w, f}(R)$-injective.

Conversely, suppose that every $\mathscr{F}_{w, f}(R)$-injective $w$-module over $R$ is injective. Let $\left\{M_{i}\right\}_{i \in I}$ be a family of $G V$-torsion-free injective $R$-modules. Then by Theorem 3.16 and [26, Proposition 2.3], $\bigoplus_{i \in I} M_{i}$ is an $\mathscr{F}_{w, f}(R)$-injective $w$-module. Therefore, by hypothesis, $\bigoplus_{i \in I} M_{i}$ is injective. Hence $R$ is $w$ Noetherian by Theorem 2.9 .

Following [13], we say that a module $M$ admits an $i$-decomposition if $M=$ $E \oplus N$, where $E$ is injective and $N$ is $i$-reduced in the sense that $N$ has no injective submodule except 0 . Note this concept was first introduced by Kaplansky. In [13, Theorem 1], it is shown that $R$ is Noetherian if and only if any $R$-module admits an $i$-decomposition. In the following, we give the $w$-analogue of this result.

Theorem 3.18. The following conditions are equivalent for a ring $R$ :

(1) $R$ is w-Noetherian;

(2) the direct sum of any countably infinite family of $G V$-torsion-free injective $R$-modules is injective;

(3) any $G V$-torsion-free $R$-module admits an i-decomposition.

Proof. $(1) \Leftrightarrow(2)$. [25, Theorem 4.4].

$(1) \Rightarrow(3)$. Let $M$ be a $G V$-torsion-free module. Set $\mathscr{S}=\{X \mid X$ is an injective submodule of $M\}$. Then $\mathscr{S}$ is nonempty since $0 \in \mathscr{S}$. Let $\left\{X_{i}\right\}$ be a chain in $\mathscr{S}$. Then by Theorem $2.9, U=\bigcup_{i} X_{i}$ is an injective submodule of $M$. Hence $U \in \mathscr{S}$ and is an upper bound of $\left\{X_{i}\right\}$. Thus there is a maximal element $E$ in $\mathscr{S}$ by Zorn's Lemma. Therefore, $M$ admits a direct decomposition $M=E \oplus N$ for some submodule $N$ of $M$. If $L$ is an injective submodule of $N$, then $E \oplus L$ is injective. By the maximality of $E$, we have $L=0$. Thus $N$ has no injective submodule except 0 . Therefore, $M$ admits an $i$-decomposition.

$(3) \Rightarrow(2)$. We adopt the proof of $\left[13\right.$, Theorem 1]. Let $\left\{M_{i}\right\}_{i=1}^{\infty}$ be a countably infinite family of $G V$-torsion-free injective $R$-modules. By hypothesis, we may assume that $I$ and $N$ realize an $i$-decomposition of $M:=\bigoplus_{i=1}^{\infty} M_{i}$. For each positive integer $n, X_{n}:=\bigoplus_{i=1}^{n} M_{i}$ is a $G V$-torsion-free injective submodule of $M$. Since $N$ is $i$-reduced, we have $E\left(X_{n} \cap I\right)=X_{n}$ from which we deduce that $X_{n} \subseteq I$. Taking direct limits on both sides gives $M=I$, which is injective.

Acknowledgements. This work was supported by the National Natural Science Foundation of China (Grant No. 11171240). The third author was supported by Basic Science Research Program through the National Research Foundation of Korea (NRF) funded by the Ministry of Education, Science and Technology (Grant No. 2010-0011996). 


\section{References}

[1] P. A. G. Asensio, M. C. Izurdiaga, and B. Torrecillas, On the existence of non-trivial finitely injective modules, Forum Math., to appear.

[2] H. Bass, Injective dimension in Noetherian rings, Trans. Amer. Math. Soc. 102 (1962), $18-29$.

[3] K. I. Beidar, S. K. Jain, and A. K. Srivastava, New characterization of $\Sigma$-injective modules, Proc. Amer. Math. Soc. 136 (2008), no. 10, 3461-3466.

[4] K. I. Beidar and W. F. Ke, On essential extensions of direct sums of injective modules, Arch. Math. (Basel) 78 (2002), no. 2, 120-123.

[5] H. Cartan and S. Eilenberg, Homological Algebra, Princeton University Press, Princeton, N. J., 1956.

[6] W. Chung, J. Ha, and H. Kim, Some remarks on strong Mori domains, Houston J. Math. 38 (2012), no. 4, 1051-1059.

[7] E. Enochs and G. Jenda, Relative Homological Algebra, Walter de Gruyter, Berlin, 2000.

[8] C. Faith and E. A. Walker, Direct sum representations of injective modules, J. Algebra 5 (1967), 203-221.

[9] L. Fuchs, Injective modules over Mori domains, Stud. Sci. Math. Hung. 40 (2005), $33-40$.

[10] L. Fuchs and L. Salce, Modules over Non-Noetherian Domains, Mathematical Surveys and Monographs 84, AMS, Providence, RI, 2001.

[11] J. M. Goursaud and J. Valette, Sur l'enveloppe injective des anneaux des groupes réguliers, Bull. Soc. Math. France 103 (1975), no. 1, 91-102.

[12] R. N. Gupta, On $f$-injective modules and semi-hereditary rings, Proc. Nat. Inst. Sci. India Part A 35 (1969), 323-328.

[13] Z.-X. He, Characterizations of Noetherian and hereditary rings, Proc. Amer. Math. Soc. 93 (1985), no. 3, 414-416.

[14] H. Kim, Module-theoretic characterizations of t-linkative domains, Comm. Algebra 36 (2008), no. 5, 1649-1670.

[15] H. Kim, E. S. Kim, and Y. S. Park, Injective modules over strong Mori domains, Houston J. Math. 34 (2008), no. 2, 349-360.

[16] H. Kim and S. Park, Module-theoretic characterizations of generalized GCD domains, III, J. Chungcheong Math. Soc., to appear.

[17] H. Kim and F. Wang, Localization of injective modules over $w$-Noetherian rings, Bull. Korean Math. Soc., to appear.

[18] R. Kurshan, Rings whose cyclic modules have finitely generated socle, J. Algebra 15 (1970), 376-386.

[19] E. Matlis, Injective modules over Noetherian rings, Pacific. J. Math. 6 (1958), 512-528.

[20] Z. Papp, On algebraically closed modules, Publ. Math. Debrecen 6 (1959), 311-327.

[21] V. S. Ramamurthi and K. M. Rangaswamy, On finitely injective modules, J. Austral. Math. Soc. 16 (1973), 239-248.

[22] F. Wang, Finitely presented type modules and w-coherent rings, J. Sichuan Normal Univ. 33 (2010), 1-9.

[23] F. Wang and R. L. McCasland, On w-modules over strong Mori domains, Comm. Algebra 25 (1997), no. 4, 1285-1306.

[24] - On strong Mori domains, J. Pure Appl. Algebra 135 (1999), no. 2, 155-165.

[25] F. Wang and J. Zhang, Injective modules over $w$-Noetherian rings, Acta Math. Sinica (Chin. Ser.) $\mathbf{5 3}$ (2010), no. 6, 1119-1130.

[26] H. Yin, F. Wang, X. Zhu, and Y. Chen, w-modules over commutative rings, J. Korean Math. Soc. 48 (2011), no. 1, 207-222. 
JUN ZHANG

Institute of Mathematics and Software Science

School of Foreign LANGUAGES

Sichuan Normal University

Chengdu 610068, P. R. China

E-mail address: zhangjun566@163.com

FANGGUI WANG

Institute of Mathematics and Software Science

Sichuan Normal University

Chengdu 610068, P. R. China

E-mail address: wangfg2004@163.com

HWANKOO KIM

DEPARTMENT OF INFORMATION SECURITY

Hoseo University

AsAN 336-795, KorEA

E-mail address: hkkim@hoseo.edu 